\title{
The Association of Targeted Gut Microbiota with Body Composition in Type 2 Diabetes Mellitus
}

\author{
Wei-Chun Hung ${ }^{1}$, Wei-Wen Hung'2, Hui-Ju Tsai ${ }^{3}$, Chen-Chia Chang ${ }^{1}$, Yi-Wen Chiu ${ }^{4,5}$, Shang-Jyh Hwang ${ }^{4,5}$,
} Mei-Chuan Kuo ${ }^{4,5}$, Szu-Chia Chen ${ }^{4,5,6,7}$, Chia-Yen Dai 6 ,8 and Yi-Chun Tsai ${ }^{4,5,6,9,10 \bowtie}$

1. Department of Microbiology and Immunology, College of Medicine, Kaohsiung Medical University, Kaohsiung, Taiwan

2. Division of Endocrinology and Metabolism, Department of Internal Medicine, Kaohsiung Medical University Hospital, Kaohsiung Medical University, Kaohsiung, Taiwan.

3. Department of Family Medicine, Kaohsiung Municipal Ta-Tung Hospital, Kaohsiung Medical University, Kaohsiung, Taiwan.

4. Division of Nephrology, Department of Internal Medicine, Kaohsiung Medical University Hospital, Kaohsiung Medical University, Kaohsiung, Taiwan.

5. Faculty of Renal Care, College of Medicine, Kaohsiung Medical University, Kaohsiung, Taiwan.

6. School of Medicine, College of Medicine, Kaohsiung Medical University, Kaohsiung, Taiwan.

7. Department of Internal Medicine, Kaohsiung Municipal Hsiao-Kang Hospital, Kaohsiung Medical University, Kaohsiung, Taiwan.

8. Division of Hepatobiliary, Department of Internal Medicine, Kaohsiung Medical University Hospital, Kaohsiung Medical University, Kaohsiung, Taiwan.

9. Division of General Medicine, Department of Internal Medicine, Kaohsiung Medical University Hospital, Kaohsiung Medical University, Kaohsiung, Taiwan.

10. Cohort Research Center, Kaohsiung Medical University, Kaohsiung, Taiwan.

$\triangle$ Corresponding author: Y.-C. Tsai, Division of General Medicine, Department of Internal Medicine, Kaohsiung Medical University Hospital, Kaohsiung Medical University, Kaohsiung, Taiwan. Tel.: 8867 3121101-5029; Fax: 8867 3122810; E-mail address: lidam65@yahoo.com.tw.

(c) The author(s). This is an open access article distributed under the terms of the Creative Commons Attribution License (https://creativecommons.org/licenses/by/4.0/). See http://ivyspring.com/terms for full terms and conditions.

Received: 2020.07.27; Accepted: 2020.11.18; Published: 2021.01.01

\begin{abstract}
The association between body composition and gut microbiota in type 2 diabetes mellitus (DM) remains unknown. To elucidate the correlation of body composition and gut microbiota, we conducted a clinical study to enroll 179 patients with type 2 DM. Body composition of lean tissue index (LTI) and fat tissue index was measured by Body Composition Monitor. Eight pairs of 16S rRNA gene primers specific to Firmicutes, Bacteroidetes, the Clostridium leptum group, Bacteroides, Bifidobacterium, Akkermansia muciniphila, Escherichia coli, and Faecalibacterium prausnitzii were used to measure their abundance by quantitative polymerase chain reaction. The results showed that type 2 DM with higher abundance of phylum Firmicutes and a higher ratio of phyla Firmicutes to Bacteroidetes (phyla F/B ratio) had higher LTI. This significant correlation between phyla F/B ratio and LTI was especially evident in type 2 DM with high body mass index, and independent of glycemic control or dipeptidyl peptidase-4 inhibitor usage. In conclusion, our study demonstrated the positive association of LTI with the abundance of phylum Firmicutes and the phyla F/B ratio in type 2 DM.
\end{abstract}

Key words: Type 2 diabetes mellitus; gut microbiota; lean tissue index; Firmicutes; Bacteroidetes

\section{Introduction}

Type 2 diabetes mellitus (DM) is a major health issue worldwide. The prevalence of type $2 \mathrm{DM}$ is rapidly increasing globally, and is predicted to reach 629 million among those aged $20-79$ years by 2045 [1]. Increasing evidence has demonstrated that disruption of gut microbiota greatly contributes to the development of type $2 \mathrm{DM}[2,3]$.

Gut microbiota, trillions of microorganisms dwelling in the human gut, and weighing about 1.5 $\mathrm{kg}$, has been regarded as a microbial organ with physical functions and a salient contributor to human health and disease [4]. Dysbiosis, which has been demonstrated to provoke compositional changes of gut microbiota, alter the permeability of the intestinal barrier, and enhance metabolic endotoxin secretion, has been proved to contribute to many diseases, including obesity and type 2 DM [2,3]. Phyla Firmicutes and Bacteroidetes, together accounting for more than $80 \%$ of total gut microbiota, are subdivided into more than 100 bacterial species [5]. Although the importance of the ratio of phyla Firmicutes to Bacteroidetes (phyla F/B ratio) is still inconclusive [6], the study by Larsen et al. using real-time quantitative polymerase chain reaction ( $\mathrm{qPCR}$ ) reported that the proportion of Firmicutes is significantly decreased in patients with type $2 \mathrm{DM}$ compared to normal 
individuals, and the phyla F/B ratios is negatively and significantly correlated with plasma glucose concentration [7].

Previous studies demonstrated that butyrate, which is digested from dietary fibers by certain bacterial species, maintains a tight junction and intestinal barrier function to reduce inflammation and improves insulin sensitivity [8-10]. Faecalibacterium prausnitzii, which belongs to the Clostridium leptum group and also the Firmicutes phylum, makes a significant contribution to butyrate production [11]. In patients with type $2 \mathrm{DM}$, the abundance of $F$. prausnitzii is decreased [12-14]. Consequently, Bifidobacterium species, which display metabolic cross-feeding with butyrate-producing bacteria, are proportionally decreased in patients with type $2 \mathrm{DM}$ [15]. Recently, accumulating evidence shows Akkermansia muciniphila may improve glucose intolerance and adipose tissue inflammation, thus playing a role prior to the onset of type 2 DM [16,17]. The gut microbiota also disrupts glucose metabolism and energy homeostasis. The phylum Proteobacteria produces lipopolysaccharide (LPS), which results in low-grade inflammation and increases intestinal permeability, leading to decreased insulin sensitivity $[18,19]$. Increased abundance of Escherichia coli belonging to the Proteobacteria phylum has been reported in Chinese patients with type 2 DM [13].

Body composition, including muscle tissue, fat tissue, and the pattern of their distribution in the body, can reflect the health status and serve as the cause or consequence of complications in patients with type 2 DM $[20,21]$. Previous study demonstrated that obese individuals with type $2 \mathrm{DM}$ have different gut microbiome composition compared with those without type 2 DM [22]. However, to our best knowledge, there is no reported study addressing the association of gut microbiota and body composition in patients with type $2 \mathrm{DM}$. Therefore, the aim of this study was to investigate the relationship between body composition and eight taxonomic units of which abundances have been reported to be related to type 2 DM, including the phyla Firmicutes and Bacteroidetes, the C. leptum group, genera Bacteroides, Bifidobacterium, A. muciniphila, E. coli, and F. prausnitzii, in patients with type $2 \mathrm{DM}$.

\section{Materials and Methods}

\section{Study participants}

This observational study was conducted in the outpatient department of a tertiary hospital in southern Taiwan from October 2016 to August 2017. Patients with antibiotic use in less than one month prior to enrollment, or with inflammatory bowel disease or with surgery of the gastrointestinal tract, or with currently diagnosed cancer undergoing chemotherapy in the previous year were excluded. Finally, we included 179 patients with type $2 \mathrm{DM}$ in this study. The study protocol was approved by the Institutional Review Board of Kaohsiung Medical University Hospital (KMUHIRB-G(II)-20160021). Informed consent was obtained in written form from all of the patients, and all clinical investigations were conducted according to the principles expressed in the Declaration of Helsinki.

\section{Sample and clinical data collection}

Diabetes was defined using blood glucose values based on American Diabetes Association criteria, a history of diabetes, or the use of anti-diabetic agents [23]. Demographic statistics, including history of cigarette smoking and alcohol drinking, and clinical data were obtained from interviews with the patients and medical records at enrollment. Hypertension was defined as a history of hypertension or the use of antihypertensive drugs. Information on the use of medications, including anti-diabetic agents, statins, and anti-hypertensive agents, was obtained at enrollment from medical records. Body mass index (BMI) was calculated as body weight in kilograms divided by body height in squared meters. All study participants were enrolled in the diabetic education program and the principles of diet therapy to diabetes were delivered on individual basis. We recorded usual diet habits in these patients by a simple questionnaire as listed in Table 1. The patients were asked to fast for at least 12 hours before blood was taken for biochemical study.

\section{Measurement of body composition}

Body composition was measured once using a bioimpedance spectroscopy method, Body Composition Monitor (BCM, Fresenius Medical Care, Germany) at the same day of collecting blood samples. The BCM measures the impedance spectroscopy at 50 different frequencies from $5 \mathrm{kHz}$ to $1 \mathrm{MHz}$, and has been validated against gold-standard methods in the general population [24,25]. Patients had been in the recumbent position for at least 5 minutes, and then electrodes were attached to one hand and one foot on the ipsilateral side. The measurement results were optimized, and raw data was adapted to the model function. The quality of the raw data was displayed as the value $Q$, with values near 100 representing high data quality and near 0 representing low data quality. Only the parameters for which the quality of the measurement was $95 \%$ or more were included in the analysis. The BCM can distinguish muscle mass and fat mass from pathologic 
fluid retention in the body based on the difference of impedance in each tissue through a new 3-component tissue-based model [26] and provide information including normohydrated lean tissue, normohydrated adipose tissue, and excess fluid mass [27]. Normohydrated lean tissue and normohydrated adipose tissue were presented as lean tissue index (LTI, lean tissue mass/height ${ }^{2}$ ) and fat tissue index (FTI, adipose tissue mass/height ${ }^{2}$ ), respectively [26]. The normal ranges of LTI and FTI are $12-17 \mathrm{~kg} / \mathrm{m}^{2}$ and $4.25-10.5 \mathrm{~kg} / \mathrm{m}^{2}$ respectively. The output parameters fit into the same reference ranges set by Fresenius Medical Care (Germany) [26,28].

Table 1. The clinical characteristics of study subjects

\begin{tabular}{|c|c|}
\hline & Entire cohort $\mathrm{N}=179$ \\
\hline Age, year & $63.2 \pm 10.2$ \\
\hline Sex (male), \% & 55.3 \\
\hline Smoke, \% & 21.8 \\
\hline Alcohol, \% & 16.2 \\
\hline Cardiovascular disease, $\%$ & 16.2 \\
\hline Hypertension, \% & 66.5 \\
\hline Hyperlipidemia, \% & 82.7 \\
\hline Gout, \% & 7.8 \\
\hline BMI, $\mathrm{kg} / \mathrm{m}^{2}$ & $26.3 \pm 4.0$ \\
\hline Mean arterial pressure, $\mathrm{mmHg}$ & $98.2 \pm 11.6$ \\
\hline Diabetic duration, years & $10.0(5.0,15.0)$ \\
\hline $\mathrm{LTI}, \mathrm{kg} / \mathrm{m}^{2}$ & $11.8 \pm 2.3$ \\
\hline FTI, $\mathrm{kg} / \mathrm{m}^{2}$ & $14.1 \pm 4.4$ \\
\hline \multicolumn{2}{|l|}{ Diet habit, $\%$} \\
\hline Protein more than fiber & 13.1 \\
\hline Fiber more than protein & 32.7 \\
\hline Fiber equal to protein & 54.2 \\
\hline \multicolumn{2}{|l|}{ Medications } \\
\hline Novonorm user, $\%$ & 5.0 \\
\hline Sulfonylurea user, \% & 46.4 \\
\hline DPP4-inhibitor user, \% & 61.5 \\
\hline Metformin user, $\%$ & 81.6 \\
\hline Insulin user, $\%$ & 15.1 \\
\hline Statin user, \% & 49.7 \\
\hline \multicolumn{2}{|l|}{ Microbiota } \\
\hline Firmicutes, copies $\times 10^{9} / \mathrm{g}$ & $5.31(2.38,8.65)$ \\
\hline Bacteroidetes, copies $\times 10^{9} / \mathrm{g}$ & $10.16(4.39,19.94)$ \\
\hline Firmicutes/Bacteroidetes & $0.54(0.24,1.10)$ \\
\hline C. leptum group, copies $\times 10^{8} / \mathrm{g}$ & $7.69(2.64,13.37)$ \\
\hline Bacteroides, copies $\times 10^{9} / \mathrm{g}$ & $2.04(0.94,4.34)$ \\
\hline Bifidobacterium, copies $\times 10^{6} / \mathrm{g}$ & $1.77(0.19,10.65)$ \\
\hline A. muciniphila, copies $\times 10^{5} / \mathrm{g}$ & $0.15(0.05,175.52)$ \\
\hline E. coli, copies $\times 10^{8} / \mathrm{g}$ & $1.23(0.34,5.82)$ \\
\hline F. prausnitzii, copies $\times 10^{7} / \mathrm{g}$ & $10.69(2.25,31.40)$ \\
\hline \multicolumn{2}{|l|}{ Laboratory parameters } \\
\hline $\mathrm{HbA1c}, \%$ & $6.9(6.5,7.9)$ \\
\hline Creatinine, mg/dl & $0.9(0.7,1.11)$ \\
\hline Hemoglobin, g/dl & $13.9 \pm 7.5$ \\
\hline Albumin, g/dl & $4.6(4.3,4.7)$ \\
\hline Calcium, mg/dl & $9.3 \pm 0.4$ \\
\hline Phosphate, mg/dl & $3.7(3.3,4.0)$ \\
\hline Uric acid, mg/dl & $5.9 \pm 1.5$ \\
\hline Cholesterol, mg/dl & $165(144,191)$ \\
\hline Triglyceride, mg/dl & $117(83,180)$ \\
\hline
\end{tabular}

Stool sample collection and microbial DNA extraction

Fecal samples were collected by patients at home, immediately frozen in the household freezer, and brought to the hospital within 12 hours. The time of fecal sample collection was either the evening one day before or the morning on the same day of obtaining biochemical data. Then, fecal samples were transferred to the laboratory and stored at $-80{ }^{\circ} \mathrm{C}$ for up to three days before processing. Bacterial DNA was extracted using the Stool DNA Extraction kit (Topgen Biotechnology Co., Ltd, Kaohsiung, Taiwan). In brief, the fecal samples were weighed to $50-100 \mathrm{mg}$ and were supplemented with a preceding bead beating (45 seconds; speed: 3450 oscillations/min). The subsequent steps of DNA extraction were performed according to the manufacturer's protocol. DNA concentration and quality was assessed by Colibri Microvolume spectrophotometer (Titertek Berthold, Pforzheim, Germany). Extracted DNA samples were immediately stored at $-20^{\circ} \mathrm{C}$ before use.

\section{Real-time quantitative polymerase chain reaction (qPCR)}

Real-time $\mathrm{QPCR}$ was utilized to measure bacterial 16S rRNA gene copies in feces in the StepOnePlus Real-Time PCR system (Thermo Fisher Scientific, Waltham, MA, USA) as in a previous study [29]. Eight pairs of 16S rRNA gene primers specific to Firmicutes, Bacteroidetes, the C. leptum group, Bacteroides, Bifidobacterium, A. muciniphila, E. coli, and F. prausnitzii are listed in Supplementary Table 1. Standard curves were constructed with a 10-fold dilution series of the 16S rRNA gene fragment amplified from the reference strains that was cloned into a T\& $\mathrm{A}^{\mathrm{TM}}$ Cloning Vector (Yeastern Biotech, Co., Ltd, Taipei, Taiwan). Each reaction mixture with a total volume of $10 \mu \mathrm{l}$ was composed of $0.25 \mu \mathrm{l}$ of each $10 \mu \mathrm{M}$ primer, $5 \mu \mathrm{l}$ of AceQ qPCR SYBR Green Master Mix (Vazyme Biotech Co., Piscataway, NJ, USA), $1 \mu \mathrm{l}$ of sample DNA, and $3.5 \mu \mathrm{l}$ sterilized ultra-pure water. Real-time PCR was carried out by the following cycle conditions: an initial holding at $95{ }^{\circ} \mathrm{C}$ for $30 \mathrm{~s}$, followed by 40 cycles of denaturation at $95^{\circ} \mathrm{C}$ for $3 \mathrm{~s}$, then annealing/elongation at $60^{\circ} \mathrm{C}$ for $40 \mathrm{~s}$. Melting curve analysis was performed after amplification to determine the specificity. Quantitation of the eight taxonomic units was evaluated as the copy numbers of the 16S rRNA genes/gram of feces weight. All qPCR tests were performed in duplicate, and the presented data are the mean values of duplicate qPCR analysis. 


\section{Statistical Analysis}

Continuous variables are presented with mean \pm SD or median $\left(25^{\text {th }}, 75^{\text {th }}\right.$ percentile), and those with skewed distribution were log-transformed to approximate a normal distribution. Categorical variables are presented as percentages. The significance of differences in continuous variables between groups was analyzed using the KruskalWallis $\mathrm{H}$ test. The chi-squared test was utilized to test differences in the distribution of categorical variables. Linear regression was used to evaluate the determinants of body composition in the patients with type $2 \mathrm{DM}$. All the variables in Table 1 tested by univariate analysis and those variables with $p$ value less than 0.05, age, and sex were selected in a multivariate linear regression analysis. Statistical analyses were conducted using SPSS version 18.0 for Windows (SPSS Inc., Chicago, Illinois) and graphs were drawn using Graph Pad Prism 5.0 (GraphPad Software Inc., San Diego CA, USA). Statistical significance was set at a two-sided $p$ value of $<0.05$.

\section{Results}

\section{Characteristics of the entire cohort}

Table 1 reveals the clinical characteristics, medication records, microbial abundance, and laboratory parameters of the entire cohort. Of the 179 subjects, the mean age was $63.2 \pm 10.2$ years, $55.3 \%$ were male, and the median of diabetic duration was $10.0(5.0,15.0)$ years. The prevalence of hypertension and hyperlipidemia was $66.5 \%$ and $82.7 \%$, respectively. The means of BMI, $\mathrm{LTI}$, and FTI were $26.3 \pm 4.0,11.8 \pm 2.3$, and $14.1 \pm 4.4 \mathrm{~kg} / \mathrm{m}^{2}$, respectively. The median of $\mathrm{HbA} 1 \mathrm{c}$ was $6.9 \%$ among study subjects.

\section{Gut microbiota and LTI in the patients with type 2 DM}

The medium of bacterial concentrations (estimated as copy numbers of $16 \mathrm{~S}$ rDNA per gram of feces) are listed in Table 1. The positive associations of LTI with the phylum Firmicutes (Spearman's rho = $0.213, p$-value $=0.004)$ and phyla $\mathrm{F} / \mathrm{B}$ ratio (Spearman's rho $=0.239, p$-value $=0.001$ ) were shown in the patients with type 2 DM (Table 2). Because of abnormal distribution of gut microbiota amount, we stratified type 2 diabetic patients according to tertiles of LTI $\left(10.6\right.$ and $\left.12.7 \mathrm{~kg} / \mathrm{m}^{2}\right)$ to precisely analyze the distribution of gut microbiota among these patients with different level of LTI (Table 3). The stepwise increase in the abundance of the phylum Firmicutes and the F/B ratio was found from tertile 1 to tertile 3 . There was a significant difference in the abundance of Bacteroides and A. muciniphila among LTI tertiles. However, no stepwise increase in the abundance of Bacteroides and A. muciniphila was shown from tertile 1 to tertile 3 in the patients with type $2 \mathrm{DM}$. There was no difference in the abundance of Bacteroidetes, Bifidobacterium, E. coli, or F. prausnitzii in the patients with different LTIs. We also stratified these patients based on LTI of median to evaluate the distribution of gut microbiota and the results were similar with our current results (Supplementary Table 2).

Table 2. The correlation among microbiome, lean tissue index, fat tissue index and body mass index in study subjects

\begin{tabular}{|c|c|c|c|c|c|c|c|c|c|c|}
\hline & & $\begin{array}{l}\text { Firmicutes } \\
\text { (copies/g) }\end{array}$ & $\begin{array}{l}\text { Bacteroidetes } \\
\text { (copies/g) }\end{array}$ & $\begin{array}{l}\text { Phyla F/B } \\
\text { ratio }\end{array}$ & $\begin{array}{l}\text { C. leptum group } \\
\text { (copies/g) }\end{array}$ & $\begin{array}{l}\text { Bacteroides } \\
\text { (copies/g) }\end{array}$ & $\begin{array}{l}\text { Bifidobacterium } \\
\text { (copies/g) }\end{array}$ & $\begin{array}{l}\text { A. muciniphila } \\
\text { (copies/g) }\end{array}$ & $\begin{array}{l}\text { E. coli } \\
\text { (copies/g) }\end{array}$ & $\begin{array}{l}\text { F. prausnitzii } \\
\text { (copies } / g \text { ) }\end{array}$ \\
\hline LTI & Spearman's rho & 0.213 & -0.077 & 0.239 & 0.018 & -0.068 & 0.084 & -0.118 & 0.052 & -0.038 \\
\hline$\left(\mathrm{Kg} / \mathrm{m}^{2}\right)$ & $P$ value & 0.004 & 0.307 & 0.001 & 0.808 & 0.367 & 0.262 & 0.116 & 0.49 & 0.618 \\
\hline FTI & Spearman's rho & -0.127 & -0.043 & -0.067 & -0.047 & -0.038 & -0.112 & -0.026 & 0.018 & -0.087 \\
\hline$\left(\mathrm{Kg} / \mathrm{m}^{2}\right)$ & $P$ value & 0.09 & 0.568 & 0.373 & 0.535 & 0.616 & 0.137 & 0.727 & 0.809 & 0.246 \\
\hline BMI & Spearman's rho & -0.021 & -0.075 & 0.042 & -0.028 & -0.085 & -0.08 & -0.063 & 0.063 & -0.123 \\
\hline$\left(\mathrm{Kg} / \mathrm{m}^{2}\right)$ & $P$ value & 0.784 & 0.318 & 0.579 & 0.714 & 0.259 & 0.285 & 0.405 & 0.401 & 0.1 \\
\hline
\end{tabular}

Abbreviations: BMI, body mass index; FTI, fat tissue index; LTI, lean tissue index.

Table 3. The microbiota of the study subjects stratified by lean tissue index

\begin{tabular}{|c|c|c|c|c|}
\hline Lean tissue index & Tertile $1(\mathrm{~N}=60)$ & Tertile $2(\mathrm{~N}=60)$ & Tertile $3(\mathrm{~N}=59)$ & $P$ value \\
\hline Firmicutes, copies $\times 10^{9} / \mathrm{g}$ & $3.82(2.21,5.97)$ & $5.91(2.77,10.30)$ & $5.91(2.82,9.67)$ & 0.03 \\
\hline Bacteroidetes, copies $\times 10^{9} / \mathrm{g}$ & $10.22(4.60,24.73)$ & $12.81(6.58,23.54)$ & $8.24(3.78,14.92)$ & 0.06 \\
\hline Phyla F/B ratio & $0.38(0.15,0.75)$ & $0.51(0.28,0.92)$ & $0.69(0.37,1.85)$ & 0.004 \\
\hline C. leptum group copies $\times 10^{8} / \mathrm{g}$ & $6.87(2.20,13.30)$ & $8.64(4.33,14.92)$ & $6.05(2.26,13.52)$ & 0.30 \\
\hline Bacteroides, copies $\times 10^{9} / \mathrm{g}$ & $2.04(0.95,5.39)$ & $2.72(1.36,4.71)$ & $1.47(0.55,2.82)$ & 0.03 \\
\hline Bifidobacterium, copies $\times 10^{6} / \mathrm{g}$ & $1.76(0.49,11.73)$ & $1.87(0.13,10.76)$ & $2.05(0.58,10.64)$ & 0.67 \\
\hline A. muciniphila, copies $\times 10^{5} / \mathrm{g}$ & $0.17(0.05,459.78)$ & $0.38(0.07,657.71)$ & $0.09(0.03,9.92)$ & 0.02 \\
\hline E. coli, copies $\times 10^{8} / \mathrm{g}$ & $1.04(0.31,6.37)$ & $2.60(0.55,7.81)$ & $1.16(0.27,4.29)$ & 0.22 \\
\hline F. prausnitzii, copies $\times 10^{7} / \mathrm{g}$ & $8.89(1.70,25.80)$ & $15.98(3.20,36.19)$ & $8.43(2.37,31.45)$ & 0.45 \\
\hline
\end{tabular}


Table 4. The determinants of lean tissue index in study subjects

\begin{tabular}{|c|c|c|c|c|c|c|}
\hline & \multicolumn{2}{|l|}{ Univariate } & \multicolumn{2}{|l|}{ Multivariate (stepwise) } & \multicolumn{2}{|c|}{ Multivariate (stepwise) } \\
\hline & $\beta(95 \% \mathrm{Cl})$ & $P$ value & $\beta(95 \% \mathrm{Cl})$ & $P$ value & $\beta(95 \% \mathrm{Cl})$ & $P$ value \\
\hline \multicolumn{7}{|l|}{ Clinical characteristics } \\
\hline Age, year & $-0.06(-0.10,-0.03)$ & $<0.001$ & $-0.06(-0.08,-0.03)$ & $<0.001$ & $-0.06(-0.08,-0.03)$ & $<0.001$ \\
\hline Sex (male), $\%$ & $3.08(2.58,3.58)$ & $<0.001$ & $2.95(2.47,3.43)$ & $<0.001$ & $2.98(2.51,3.45)$ & $<0.001$ \\
\hline Heart disease, $\%$ & $0.19(-0.73,1.10)$ & 0.69 & -- & -- & -- & -- \\
\hline Body mass index, $\mathrm{kg} / \mathrm{m}^{2}$ & $0.12(0.04,0.20)$ & 0.005 & -- & -- & -- & -- \\
\hline Mean arterial pressure, $\mathrm{mmHg}$ & $0.00(-0.03,0.03)$ & 0.82 & -- & -- & -- & -- \\
\hline \multicolumn{7}{|l|}{ Diet habit, \% } \\
\hline Protein more than fiber & $-0.71(-1.94,0.53)$ & 0.26 & & & & \\
\hline Fiber more than protein & $0.56(-0.33,1.45)$ & 0.21 & & & & \\
\hline \multicolumn{7}{|l|}{ Microbiome (log-formed) } \\
\hline Firmicutes, copies* $10^{9} / \mathrm{g}$ & $1.01(0.20,1.82)$ & 0.02 & $0.65(0.06,1.23)$ & 0.03 & -- & -- \\
\hline Bacteroidetes, copies ${ }^{*} 0^{9} / \mathrm{g}$ & $-0.08(-0.59,0.42)$ & 0.75 & -- & -- & -- & -- \\
\hline Firmicutes/Bacteroidetes & $1.06(0.43,1.70)$ & 0.001 & -- & -- & $0.48(0.02,0.94)$ & 0.04 \\
\hline C. leptum group, copies* $10^{8} / \mathrm{g}$ & $-0.02(-0.67,0.62)$ & 0.94 & -- & -- & -- & -- \\
\hline Bacteroides, copies* $10^{8} / \mathrm{g}$ & $-0.10(-0.60,0.41)$ & 0.7 & -- & -- & -- & -- \\
\hline Bifidobacterium, copies*106/g & $0.14(-0.09,0.38)$ & 0.23 & -- & -- & -- & -- \\
\hline A. muciniphila, copies* $10^{5} / \mathrm{g}$ & $-0.14(-0.30,0.02)$ & 0.08 & -- & -- & -- & -- \\
\hline E. coli, copies* $10^{8} / \mathrm{g}$ & $-0.14(-0.48,0.20)$ & 0.42 & -- & -- & -- & -- \\
\hline F. prausnitzii, copies* $10^{7} / \mathrm{g}$ & $0.09(-0.20,0.38)$ & 0.52 & -- & -- & -- & -- \\
\hline \multicolumn{7}{|l|}{ Laboratory data } \\
\hline Log-formed $\mathrm{HbA1c}$ & $-0.10(-0.31,3.00)$ & 0.95 & -- & -- & -- & -- \\
\hline Log-formed creatinine & $3.49(1.53,5.44)$ & 0.001 & -- & -- & -- & -- \\
\hline Hemoglobin, g/dl & $0.47(0.29,0.66)$ & $<0.001$ & -- & -- & -- & -- \\
\hline Log-formed albumin & $9.83(0.94,18.71)$ & 0.03 & -- & -- & -- & -- \\
\hline Calcium, mg/dl & $0.90(-1.80,0.01)$ & 0.05 & -- & -- & -- & -- \\
\hline Log-formed phosphate & $-5.26(-9.96,-0.57)$ & 0.03 & -- & -- & -- & -- \\
\hline Uric acid, mg/dl & $0.08(-0.15,0.31)$ & 0.49 & -- & -- & -- & -- \\
\hline Log-formed cholesterol & $-3.18(-6.63,0.28)$ & 0.07 & -- & -- & -- & -- \\
\hline Log-formed triglyceride & $0.80(-0.60,2.19)$ & 0.26 & -- & -- & -- & -- \\
\hline
\end{tabular}

We performed linear regression analysis to analyze the determinants of LTI (Table 4). In univariate analysis, male, BMI, serum hemoglobin, creatinine and log-formed albumin levels, phylum Firmicutes, and phyla F/B ratio were significantly and positively associated with LTI in the patients with type 2 DM. Age and log-formed serum phosphate level were negatively correlated with LTI. Further multivariate analysis revealed that the patients with higher abundance of phylum Firmicutes $(\beta=0.65$, $95 \%$ confidence index $(\mathrm{CI})=0.06-1.23)$ and higher phyla $\mathrm{F} / \mathrm{B}$ ratio $(\beta=0.48,95 \% \mathrm{CI}=0.02-0.94)$ had higher LTI. However, there was no significant correlation between gut microbiota and FTI in the patients with type 2 DM (Table 2 and Supplementary Table 3).

\section{Gut microbiota and LTI of the patients with type 2 DM in different subgroups}

In order to investigate the effect of age, sex, glycemic control, and anti-diabetic agents usage on LTI, we also stratified the patients with type $2 \mathrm{DM}$ by age (65 years-old as cut-off value for old age), sex, $\mathrm{HbA} 1 \mathrm{c}$ (7\% as an index for optimal glycemic control), and anti-diabetic agents (Figure 1), and the results revealed a positive association of LTI with the phylum Firmicutes in the patients with age $\geq 65$ years-old, HbA1c $\leq 7 \%$, or using sulfonylurea and dipeptidyl peptidase-4 (DPP4) inhibitors. The correlation between LTI with the phyla F/B ratio was found in the patients with male gender, or using sulfonylurea. The correlation between LTI with the phyla F/B ratio was independent of age, HbA1c level, and using DPP4 inhibitors in the patients with type $2 \mathrm{DM}$. We also stratified the patients with type 2 DM by BMI (24 $\mathrm{kg} / \mathrm{m}^{2}$ as cut-off value for definition of overweight in Taiwanese population [30] (Figure 1), and found a positive correlation between LTI and the phylum Firmicutes and the phyla F/B ratio in the patients with BMI $\geq 24 \mathrm{~kg} / \mathrm{m}^{2}$, not in those with $\mathrm{BMI}<24$ $\mathrm{kg} / \mathrm{m}^{2}$.

\section{Discussion}

This is the first study to evaluate the association of the gut microbiota with body composition in the patients with type $2 \mathrm{DM}$. We found that the phylum Firmicutes and the phyla F/B ratio were significantly and positively correlated with LTI. The patients with higher abundance of Firmicutes and a higher phyla $\mathrm{F} / \mathrm{B}$ ratio were more likely to have more lean mass after adjusting for a number of variables of body composition, such as age, sex, and BMI. This significant correlation between phyla F/B ratio and LTI was especially evident in the patients with type 2 DM who had high BMI, and was independent of glycemic control or DPP4 inhibitor usage. 

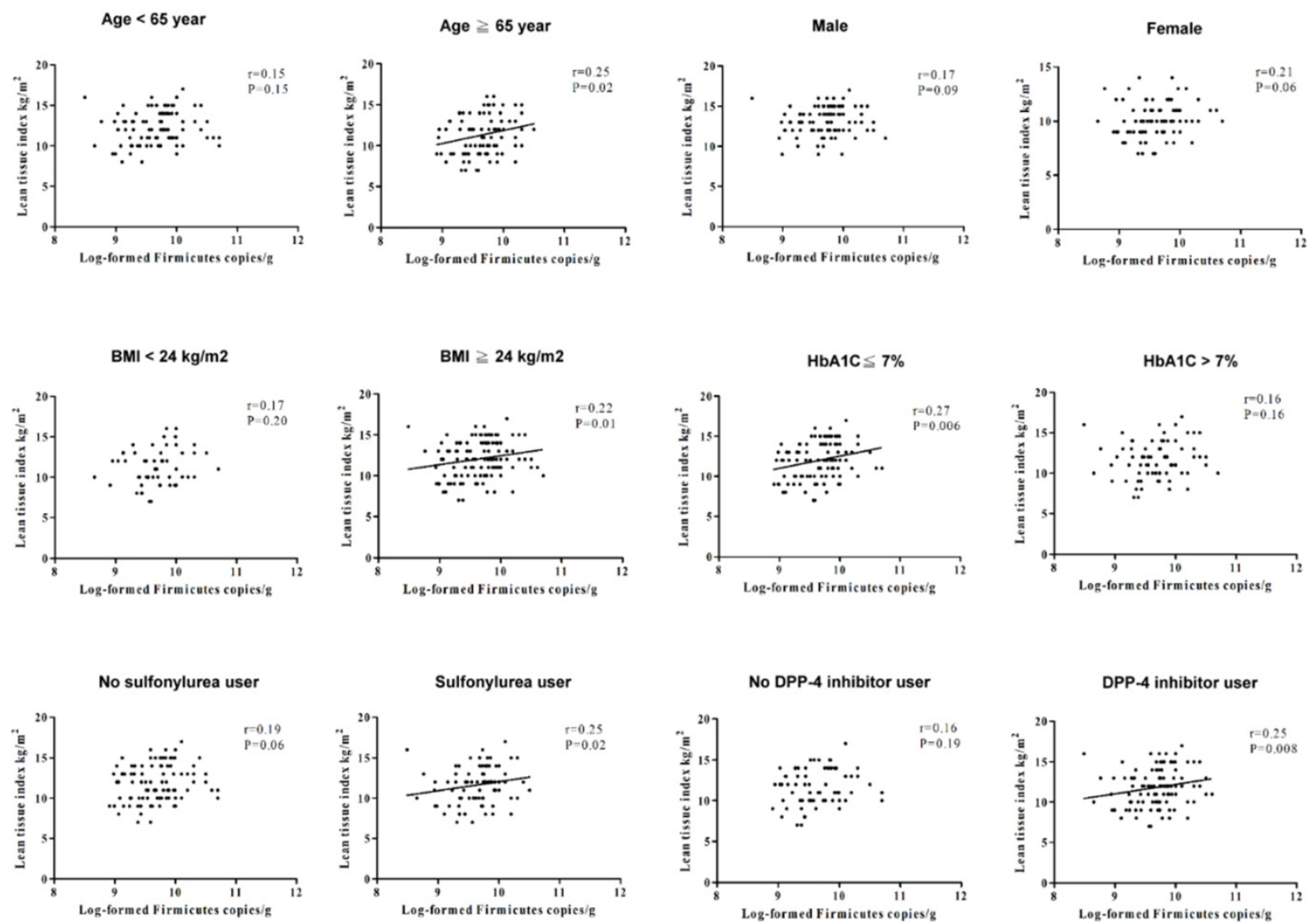

No DPP-4 inhibitor user

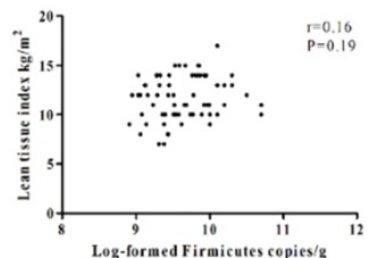

DPP-4 inhibitor user
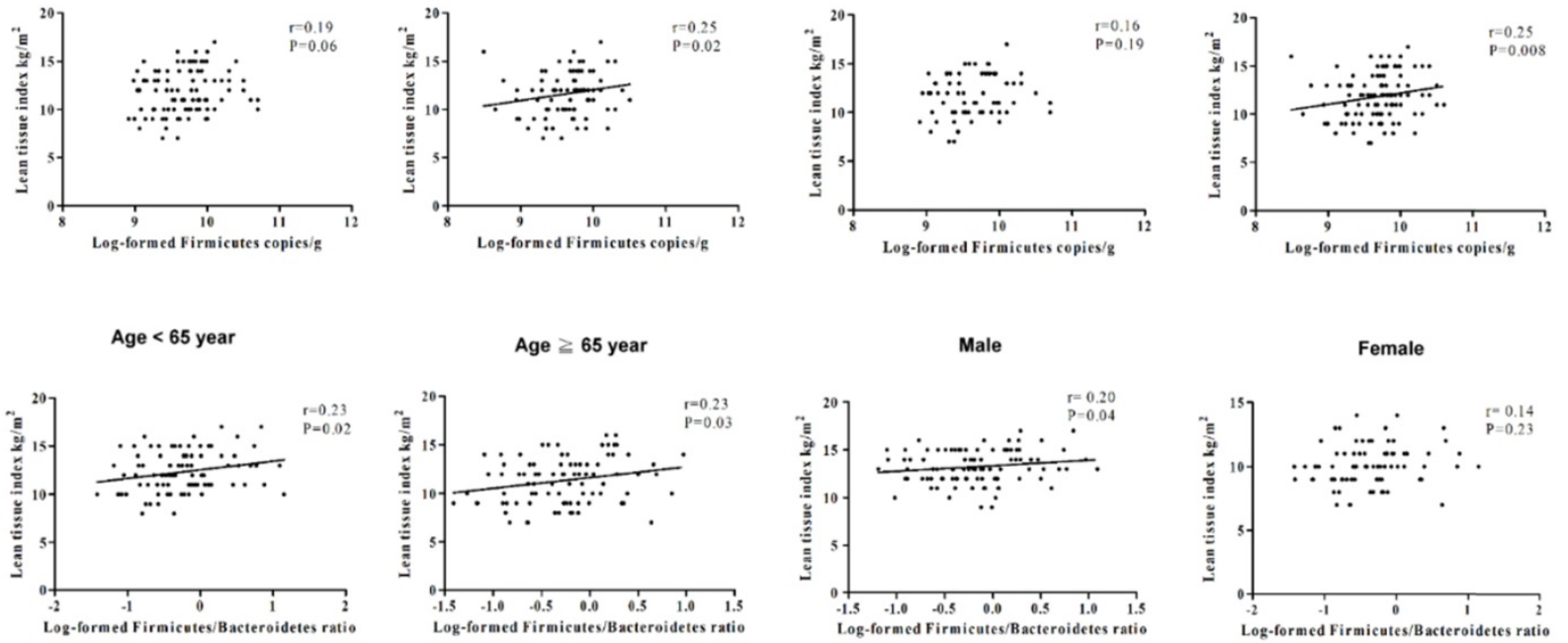

$\mathrm{BMI}<24 \mathrm{~kg} / \mathrm{m} 2$

$B M I \geqq 24 \mathrm{~kg} / \mathrm{m} 2$

HbA $1 \mathrm{C} \leq 7 \%$
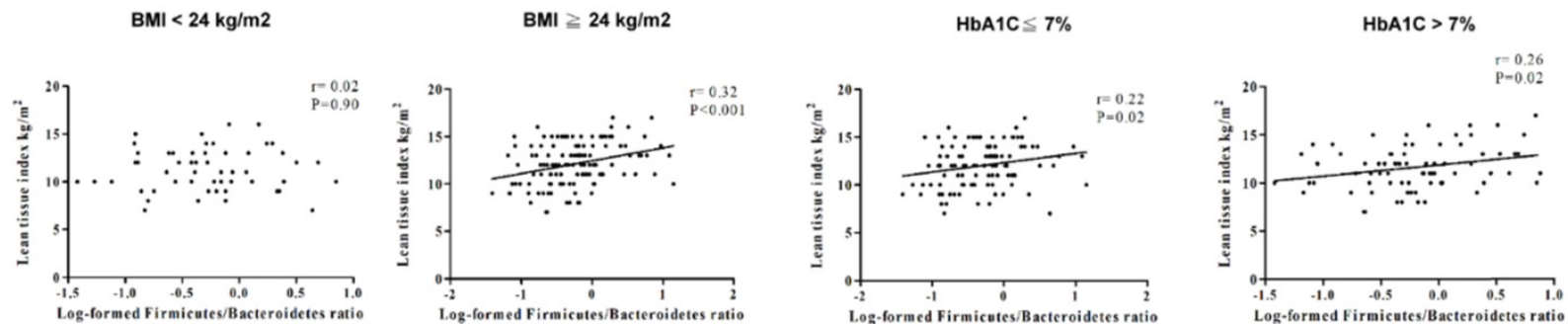

No sulfonylurea user

Sulfonylurea user

No DPP-4 inhibitor user

DPP-4 inhibitor user
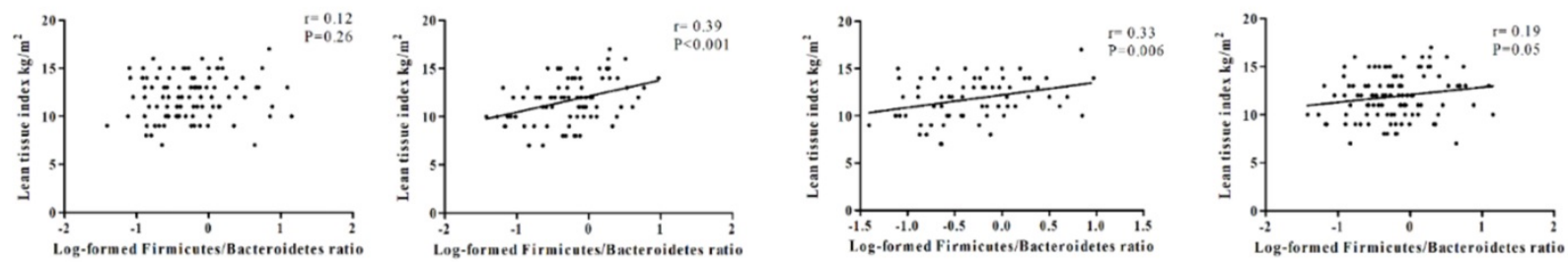

Figure 1. The correlation between lean tissue index and the phylum Firmicutes or the phyla F/B ratio in different subgroups. 
Accumulating evidence reveals that the phyla $\mathrm{F} / \mathrm{B}$ ratio is higher in obese individuals than lean individuals, and the phyla F/B ratio is positively correlated with BMI [31]. BMI has been widely used as an indicator of health. However, BMI cannot provide accurate information about the distribution of body composition. Body composition, in terms of lean tissue and fat tissue, can provide a more accurate reflection of physical function and nutrition status compared with BMI [32]. Lean mass has been presented as a nutritional marker, and is correlated with increased risks for osteoporosis, physical disability, functional impairment, hospitalization, and even mortality in the general population [33-35]. Loss of lean tissue may result in insulin resistance, inflammation, and micro- and macrovascular complications in patients with type 2 DM [36,37]. The patients with type $2 \mathrm{DM}$ usually have a lower phyla $\mathrm{F} / \mathrm{B}$ ratio than normal individuals [7]. The current results demonstrated the positive association of LTI with Firmicutes and the phyla F/B ratio in the patients with type $2 \mathrm{DM}$. The possible explanations are that Firmicutes may utilize energy sources more effectively than Bacteroidetes, and Firmicutes is correlated with the nutrient transporter, thus enhancing the lean tissue amount [18]. Furthermore, variation of LTI has been associated with all-cause mortality or cardiovascular events [38]. Based on the significant correlation between microbiota and LTI, microbiota might be the potential biomarkers to evaluate outcomes in clinical patients. Further study is needed to examine whether type 2 diabetic patients with higher abundance of Firmicutes or higher phyla $\mathrm{F} / \mathrm{B}$ ratio have better clinical outcomes than those with lower abundance of Firmicutes or lower phyla $\mathrm{F} / \mathrm{B}$ ratio.

Grosicki et al. suggest that the gut microbiota plays a principle role in the metabolism of lean tissue [18]. Previous study demonstrated that butyrateproducing bacteria (the C. leptum group and $F$. prausnitzii in this study) may reduce inflammation, leading to enhanced muscle function $[8,9]$. Bifidobacterium may affect gut-muscle communication and modulate muscle size, and supplementation of Bifidobacterium probably reduces muscle wasting [18]. The phylum Proteobacteria produces LPS and increases intestinal permeability, triggering systemic inflammation and muscle maladaptation [18]. However, our results did not find an association of LTI with the C. leptum group, F. prausnitzii, Bifidobacterium, or E.coli in the patients with type 2 DM. This inconsistent finding may relate to differences in study population, race, and diet habit. It has been reported that supplementation with $A$. muciniphila slightly decreased fat mass in overweight or obese individuals [16]. Our results showed significant difference but no stepwise increase in the abundance of A. muciniphila (Table 3), which might be due to the wide distribution range of the single microbial species seen in our study with small sample size.

The gut microbiota regulates the amount, distribution, and storage of fat tissue in mice [39]. However, our results did not reveal a significant correlation between fat tissue and gut microbiota in the patients with type $2 \mathrm{DM}$. The relatively small number of the patients with type 2 DM may underestimate the effect of gut microbiota on the alteration of fat tissue. Furthermore, this study only measured eight targeted gut microbiota, and other bacteria related to fat tissue might not be examined. In addition, muscle wasting is probably one of the reasons why microbiota was correlated with LTI, and not with FTI, in the patients with type 2 DM. In accordance with the measured LTI compared with the age- and gender-normalized LTI value, an LTI less than $10 \%$ of the normal value indicates muscle wasting [40]. These patients were under stable clinical condition and had high serum albumin level $(4.7 \pm 0.2$ $\mathrm{g} / \mathrm{dl}$ ), as a nutrition marker, meaning that they might not have presented with the real status of muscle wasting. It is necessary to examine the interactional effect of muscle wasting on the correlation between microbiota and body composition in the future.

Overweightness and obesity are common in type 2 DM [41]. The composition of gut microbiota may be altered in overweight or obese patients with type 2 DM. We stratified the patients with type $2 \mathrm{DM}$ by BMI of $24 \mathrm{~kg} / \mathrm{m}^{2}$, as the cut-off value for the definition of being overweight in the Taiwanese population [30], to analyze the correlation between LTI and gut microbiota. We found a positive association of LTI with both the abundance of Firmicutes and the F/B ratio in the patients with $\mathrm{BMI} \geq 24 \mathrm{~kg} / \mathrm{m}^{2}$, but not in those with BMI $<24 \mathrm{~kg} / \mathrm{m}^{2}$. The abundance of Firmicutes and the F/ $\mathrm{B}$ ratio may influence lean tissue mass in the patients with type $2 \mathrm{DM}$, especially those with high BMI.

Type $2 \mathrm{DM}$ is associated with an altered amount and distribution of lean tissue and fat tissue [35]. Wierzbicka et al. demonstrated that the patients with type $1 \mathrm{DM}$ having lower $\mathrm{HbA1c}$ levels had elevated lean mass [42]. However, there was no correlation between body composition variables and $\mathrm{HbA} 1 \mathrm{c}$ in type 2 DM $[43,44]$. The relationship between $\mathrm{HbA1c}$ and body composition is controversial in patients with DM. In addition, plasma DPP4 activity is reported to be positively correlated with lean mass and central adiposity, and negatively with general adiposity [45]. Sulfonylurea has the well-known side 
effects of weight gain [46], which might lead to change body composition. Therefore, we further divided the patients with type $2 \mathrm{DM}$ into $\mathrm{HbA} 1 \mathrm{c} \leq 7 \%$ or $>7 \%$, and users or non-users of DPP4 inhibitors or sulfonylurea. The correlation between LTI and the phyla F/B ratio was independent of the HbA1c level and DPP4 inhibitor usage. This positive correlation was shown in sulfonylurea users of the patients with type $2 \mathrm{DM}$. The mechanism for sulfonylurea to affect body composition may be mediated by gut microbiota.

In our study, more than $80 \%$ of participants with type $2 \mathrm{DM}$ were treated with metformin, and there was no significant difference of the abundance of eight bacteria between the patients with and without metformin usage (Supplementary Table 4). Thus, metformin is not a confounder in this study. Previous literature demonstrated that metformin would induce the variation of microbiome composition [47-51]. The contradiction might be due to the experimental methods. Our study implemented qPCR to compare the eight targeted microbial species instead of analyzing the whole gut microbiome with $16 \mathrm{~S}$ rRNA gene sequencing in many other studies.

Our study disclosed for the first time that associations might exist between gut microbiota and body composition in the patients with type $2 \mathrm{DM}$. However, three are some limitations in this study. Firstly, only eight targeted gut microbiota were measured by real-time qPCR instead of by $16 \mathrm{~S}$ rRNA sequencing. Compared to $16 \mathrm{~S}$ RNA sequencing, real-time qPCR is cost-effective and time-saving and provides the ability to measure the absolute quantity rather than the relative percentage. However, without analyzing gut microbiota with the gold-standard method of $16 \mathrm{~S}$ rRNA sequencing, our results fail to present the whole microbiome signature pertaining to body composition. Further advanced study using $16 \mathrm{~S}$ rRNA sequencing would be needed to reveal the whole profile of the gut microbiota associated with body composition. Moreover, the cross-sectional design of this study does not allow demonstration of the cause-effect relationship of gut microbiota and body composition, and might even lead to random results. Future longitudinal study of gut microbiota and body composition is needed and a separate cohort study should be conducted to confirm our novel finding. Finally, although we recorded usual diet habits, the data of detailed diet content (total energy, carbohydrate, protein, and fat intake) is lacking, and might underestimate the impact of diet on the correlation between microbiota and body composition.

In conclusion, this study demonstrated for the first time the relationship between gut microbiota and body composition in the patients with type $2 \mathrm{DM}$. The abundance of Firmicutes and the phyla F/B ratio were significantly associated with lean tissue. Further study will be conducted to analyze the interaction between gut microbiota and body composition in clinical outcomes of the patients with type $2 \mathrm{DM}$.

\section{Supplementary Material}

Supplementary figures and tables. http://www.medsci.org/v18p0511s1.pdf

\section{Acknowledgements}

The authors thank the Center for Research Resources and Development, Kaohsiung Medical University, Kaohsiung, Taiwan.

\section{Funding}

This study was funded by grants from Ministry of Science and Technology (MOST 108-2635-B-037004), Kaohsiung Medical University (KMU-TC108B07, KMU-DK108004, and SA10802), Kaohsiung Medical University Hospital (KMUH106-6M11, KMUH1077M10, KMUH108-8R20, and KMUH108-8R29), and Kaohsiung Municipal Siaogang Hospital (KMHK-A107-002). The funders had no role in study design, data collection and analysis, decision to publish, or preparation of the manuscript.

\section{Author Contributions}

$\mathrm{WCH}, \mathrm{WWH}, \mathrm{HJT}$, and YCT designed the study, WWH, YWC, SJH, MCK, SCC CYD, and YCT collected the clinical data, CCC performed the experiment and collected the result, $\mathrm{WCH}, \mathrm{HJT}$, and YCT processed the statistical analysis, $\mathrm{WCH}$ drafted the manuscript, and YCT reviewed and edited the final manuscript. All authors have revised and approved the submitted manuscript.

\section{Competing Interests}

The authors have declared that no competing interest exists.

\section{References}

1. Cho NH, Shaw JE, Karuranga S, et al. IDF Diabetes Atlas: Global estimates of diabetes prevalence for 2017 and projections for 2045. Diabetes Res Clin Pract. 2018; 138: 271-81.

2. Brunkwall L, Orho-Melander $\mathrm{M}$. The gut microbiome as a target for prevention and treatment of hyperglycaemia in type 2 diabetes: from current human evidence to future possibilities. Diabetologia. 2017; 60: 943-51.

3. Pascale A, Marchesi N, Govoni S, et al. The role of gut microbiota in obesity, diabetes mellitus, and effect of metformin: new insights into old diseases. Curr Opin Pharmacol. 2019; 49: 1-5.

4. Zhao L. The gut microbiota and obesity: from correlation to causality. Nat Rev Microbiol. 2013; 11: 639-47.

5. Rajilic-Stojanovic M, de Vos WM. The first 1000 cultured species of the human gastrointestinal microbiota. FEMS Microbiol Rev. 2014; 38: 996-1047.

6. Zmora N, Suez J, Elinav E. You are what you eat: diet, health and the gut microbiota. Nat Rev Gastroenterol Hepatol. 2019; 16: 35-56.

7. Larsen N, Vogensen FK, van den Berg FW, et al. Gut microbiota in human adults with type 2 diabetes differs from non-diabetic adults. PLoS One. 2010; 5 : e9085. 
8. Peng L, Li ZR, Green RS, et al. Butyrate enhances the intestinal barrier by facilitating tight junction assembly via activation of AMP-activated protein kinase in Caco-2 cell monolayers. J Nutr. 2009; 139: 1619-25.

9. Cox MA, Jackson J, Stanton $\mathrm{M}$, et al. Short-chain fatty acids act as antiinflammatory mediators by regulating prostaglandin $\mathrm{E}(2)$ and cytokines. World J Gastroenterol. 2009; 15: 5549-57.

10. Gao Z, Yin J, Zhang J, et al. Butyrate improves insulin sensitivity and increases energy expenditure in mice. Diabetes. 2009; 58: 1509-17.

11. Riviere A, Selak M, Lantin D, et al. Bifidobacteria and butyrate-producing colon bacteria: importance and strategies for their stimulation in the human gut. Front Microbiol. 2016; 7: 979.

12. Navab-Moghadam F, Sedighi M, Khamseh ME, et al. The association of type II diabetes with gut microbiota composition. Microb Pathog. 2017; 110: 630-6.

13. Qin J, Li Y, Cai Z, et al. A metagenome-wide association study of gut microbiota in type 2 diabetes. Nature. 2012; 490: 55-60.

14. Karlsson FH, Tremaroli V, Nookaew I, et al. Gut metagenome in European women with normal, impaired and diabetic glucose control. Nature. 2013; 498: 99-103.

15. Le KA, Li Y, Xu X, et al. Alterations in fecal Lactobacillus and Bifidobacterium species in type 2 diabetic patients in Southern China population. Front Physiol. 2013; 3: 496

16. Depommier C, Everard A, Druart C, et al. Supplementation with Akkermansia muciniphila in overweight and obese human volunteers: a proof-of-concept exploratory study. Nat Med. 2019; 25: 1096-103.

17. Plovier H, Everard A, Druart C, et al. A purified membrane protein from Akkermansia muciniphila or the pasteurized bacterium improves metabolism in obese and diabetic mice. Nat Med. 2017; 23: 107-13.

18. Grosicki GJ, Fielding RA, Lustgarten MS. Gut microbiota contribute to agerelated changes in skeletal muscle size, composition, and function: biological basis for a gut-muscle axis. Calcif Tissue Int. 2018; 102: 433-42.

19. Allin KH, Nielsen T, Pedersen O. Mechanisms in endocrinology: Gut microbiota in patients with type 2 diabetes mellitus. Eur J Endocrinol. 2015; 172: R167-77.

20. Heshka S, Ruggiero A, Bray GA, et al. Altered body composition in type 2 diabetes mellitus. Int J Obes (Lond). 2008; 32: 780-7.

21. Solanki JD, Makwana AH, Mehta HB, et al. Body composition in type 2 diabetes: Change in quality and not just quantity that matters. Int J Prev Med. 2015; 6: 122.

22. Thingholm LB, Ruhlemann MC, Koch M, et al. Obese Individuals with and without Type 2 diabetes show different gut microbial functional capacity and composition. Cell Host Microbe. 2019; 26: 252-64.

23. Genuth S, Alberti KG, Bennett P, et al. Follow-up report on the diagnosis of diabetes mellitus. Diabetes Care. 2003; 26: 3160-7.

24. Wabel P, Chamney $\mathrm{P}$, Moissl $\mathrm{U}$, et al. Importance of whole-body bioimpedance spectroscopy for the management of fluid balance. Blood Purif. 2009; 27: 75-80.

25. Wieskotten $S$, Heinke $S$, Wabel $P$, et al. Bioimpedance-based identification of malnutrition using fuzzy logic. Physiol Meas. 2008; 29: 639-54

26. Wang $\mathrm{YW}$, Lin TY, Peng $\mathrm{CH}$, et al. Factors associated with decreased lean tissue index in patients with chronic kidney disease. Nutrients. 2017; 9: 434

27. Chamney PW, Wabel P, Moissl UM, et al. A whole-body model to distinguish excess fluid from the hydration of major body tissues. Am J Clin Nutr. 2007; 85: 80-9.

28. Tsai YC, Tsai JC, Chen SC, et al. Association of fluid overload with kidney disease progression in advanced CKD: a prospective cohort study. Am J Kidney Dis. 2014; 63: 68-75.

29. Karlsson CL, Onnerfalt J, Xu J, et al. The microbiota of the gut in preschool children with normal and excessive body weight. Obesity (Silver Spring). 2012; 20: 2257-61.

30. Koliada A, Syzenko G, Moseiko V, et al. Association between body mass index and Firmicutes/Bacteroidetes ratio in an adult Ukrainian population. BMC Microbiol. 2017; 17: 120.

31. Woo J, Leung J, Kwok T. BMI, body composition, and physical functioning in older adults. Obesity (Silver Spring). 2007; 15: 1886-94.

32. Dugas LR, Fuller M, Gilbert J, et al. The obese gut microbiome across the epidemiologic transition. Emerg Themes Epidemiol. 2016; 13: 2.

33. Janssen I, Heymsfield SB, Ross R. Low relative skeletal muscle mass (sarcopenia) in older persons is associated with functional impairment and physical disability. J Am Geriatr Soc. 2002; 50: 889-96.

34. Metter EJ, Talbot LA, Schrager M, et al. Skeletal muscle strength as a predictor of all-cause mortality in healthy men. J Gerontol A Biol Sci Med Sci. 2002; 57: B359-65.

35. Atlantis E, Martin SA, Haren MT, et. al. Inverse associations between muscle mass, strength, and the metabolic syndrome. Metabolism. 2009; 58: 1013-22.

36. Abbatecola AM, Olivieri F, Corsonello A, et al. Frailty and safety: the example of diabetes. Drug Saf. 2012; 35 (Suppl 1): 63-71.

37. Backhed F, Ding H, Wang T, et al. The gut microbiota as an environmental factor that regulates fat storage. Proc Natl Acad Sci U S A. 2004; 101: 15718-23.

38. Lin TY, Peng CH, Hung SC, Tarng DC. 2018. Body composition is associated with clinical outcomes in patients with non-dialysis-dependent chronic kidney disease. Kidney Int 93:733-740.

39. Rosenberger J, Kissova V, Majernikova M, et al. Body composition monitor assessing malnutrition in the hemodialysis population independently predicts mortality. J Ren Nutr. 2014; 24: 172-6.
40. Yach D, Stuckler D, Brownell KD. Epidemiologic and economic consequences of the global epidemics of obesity and diabetes. Nat Med. 2006; 12: 62-6.

41. Hsiao Y-C, Wang K, Bair M-J. Prevalence of obesity and metabolic syndrome in Aboriginals in Southeastern Taiwan-a Hospital-based study. J Intern Med Taiwan. 2011; 22: 48-56.

42. Wierzbicka E, Swiercz A, Pludowski P, et al. Skeletal status, body composition, and glycaemic control in adolescents with type 1 diabetes mellitus. J Diabetes Res. 2018; 2018: 8121634.

43. Lipsky LM, Gee B, Liu A, et al. Glycemic control and variability in association with body mass index and body composition over 18 months in youth with type 1 diabetes. Diabetes Res Clin Pract. 2016; 120: 97-103.

44. Solanki JD, Makwana AH, Mehta HB, et al. Effect of current glycemic control on qualitative body composition in sedentary ambulatory Type 2 diabetics. Niger Med J. 2016; 57: 5-9.

45. Silva Junior WS, Souza M, Nogueira Neto JF, et al. Dipeptidyl peptidase 4 activity is related to body composition, measures of adiposity, and insulin resistance in subjects with excessive adiposity and different degrees of glucose tolerance. J Diabetes Res. 2019; 2019: 5238013.

46. Sola D, Rossi L, Schianca GP, et al. Sulfonylureas and their use in clinical practice. Arch Med Sci. 2015; 11: 840-8.

47. Forslund K, Hildebrand F, Nielsen T, et al. Disentangling type 2 diabetes and metformin treatment signatures in the human gut microbiota. Nature. 2015; 528: 262-6.

48. Wu H, Esteve E, Tremaroli V, et al. Metformin alters the gut microbiome of individuals with treatment-naive type 2 diabetes, contributing to the therapeutic effects of the drug. Nat Med. 2017; 23: 850-8.

49. Zhang $\mathrm{W}, \mathrm{Xu} \mathrm{JH}, \mathrm{Yu} \mathrm{T}$, Chen QK. 2019. Effects of berberine and metformin on intestinal inflammation and gut microbiome composition in $\mathrm{db} / \mathrm{db}$ mice. Biomed Pharmacother 118:109131.

50. Xourgia E, Papazafiropoulou A, Papanas N, Melidonis A. 2019. Anti-diabetic treatment leads to changes in gut microbiome. Front Biosci (Landmark Ed) 24:688-99.

51. Vallianou NG, Stratigou T, Tsagarakis S. 2019. Metformin and gut microbiota: their interactions and their impact on diabetes. Hormones (Athens) 18:141-4. 